\title{
Uso de Técnicas de Proteção de Software em Sistemas Embarcados*
}

\author{
Lucila Maria de Souza Bento ${ }^{1}$ e Raphael Carlos Santos Machado ${ }^{1,2}$ \\ ${ }^{1}$ Instituto Nacional de Metrologia, Qualidade e Tecnologia (INMETRO) \\ Duque de Caxias, RJ - Brasil \\ ${ }^{2}$ Programa de Pós-Graduação em Ciência da Computação \\ Centro Federal de Educação Tecnológica Celso Suckow da Fonseca \\ Rio de Janeiro, RJ- Brasil \\ lucilabento@ppgi.ufrj.br, rcmachado@inmetro.gov.br
}

\begin{abstract}
Embedded systems are used worldwide in measurements of public interest and consumer, industrial, automotive, medical, commercial and military applications. The security of embedded systems has been increasingly important as these embedded systems devices manage valuable digital contents or sensitive personal data. The concern around cyber security has increased in the last years with the creation of the laws for protection of personal information, e.g., GDPR (EU General Data Protection Regulation) and LGPDP (Lei Geral de Proteção de Dados Pessoais - Brazil). In the present work, we discuss how software protection techniques can help enhance cyber security in embedded systems.
\end{abstract}

Resumo. Sistemas embarcados são usados em todo mundo em aplicações de consumo, industriais, automotivas, médicas, comerciais e militares. A segurança dos sistemas embarcados tem sido cada vez mais importante, já que esses dispositivos armazenam, processam e transmitem informações digitais valiosas ou dados pessoais sensíveis. A preocupação com segurança da informação tem aumentado nos últimos anos e motivado a criação de leis de proteção de informações pessoais, como a GDPR (EU General Data Protection Regulation) e a LGPDP (Lei Geral de Proteção de Dados Pessoais), por exemplo. Neste trabalho, discutimos como técnicas de proteção de software conhecidas na literatura podem ajudar a aumentar a segurança em sistemas embarcados, garantindo a proteção dos dados manipulados.

\section{Introdução}

$\mathrm{Na}$ atualidade, os sistemas embarcados desempenham um papel importante na indústria e na economia brasileira e, a exemplo do que acontece na Europa, onde a taxa de crescimento anual no uso de sistemas embarcados atingiu 12\% [Insider 2018, IDC 2014], o uso de tais sistemas torna-se cada vez mais popular e expressivo. No contexto da metrologia legal, o cenário de crescimento persiste, impulsionado, em grande parte, por iniciativas como a modernização das redes de transmissão de energia (smart grids) e necessidade de acesso a dados de medição em tempo real.

*Trabalho parcialmente apoiado pelo projeto SHCDCiber. 
Ao compararmos o desenvolvimento de sistemas tradicionais de automação comercial, industrial e de escritório com o desenvolvimento de sistemas embarcados, podemos observar que o último requer um elevado grau de confiabilidade, segurança e proteção. Isso se deve ao fato dos sistemas embarcados, em grande parte, permanecerem imersos em ambientes não controlados onde estão sujeitos a todo tipo de intervenção (intencional ou não intencional), e mesmo diante de qualquer adversidade devem garantir seu correto funcionamento e confidencialidade dos dados processados, armazenados e/ou transmitidos. Em muitos casos, o mau funcionamento ou a manipulação indevida de sistemas embarcados pode causar danos e prejuízos consideráveis.

Por outro lado, produzir sistemas embarcados seguros não é uma tarefa fácil, pois o espaço do projeto arquitetural a ser explorado é amplo. Além dos desafios relacionados a arquitetura de hardware do dispositivo, questões como a escolha do sistema operacional, a composição do software de aplicação e a integração entre ambos são determinantes para a segurança do dispositivo.

Estudos apontam que, em média, um bug de software pode ser encontrado em 2300 linhas de código [Chelf 2011]. Isso significa que além de preocupar-se com o correto desenvolvimento dos softwares e com os problemas inerentes a esta atividade, os fabricantes ainda precisam considerar em seus projetos modelos de ataque e técnicas a serem implementadas para impedir (ou, ao menos, reduzir a probabilidade) que tais ataques ocorram. Estas e outras questões, estão tornando o tema segurança e proteção de software um dos principais pontos de atenção no projeto de um sistema embarcado seguro e um dos principais assuntos de pesquisa da atualidade.

No Brasil, a segurança de alguns tipos de sistemas embarcados é assegurada por instituições nacionais, como o Instituto Nacional de Metrologia Qualidade e Tecnologia INMETRO, que são responsáveis pela validação destes dispositivos — ou regulamentação e/ou apoio as instituições responsáveis por sua análise — antes de serem disponibilizados ao mercado consumidor. Os testes para validação dos sistemas embarcados são realizados com base em requisitos legais elaborados por tais instituições, nos quais são abordados testes de hardware e software. Entretanto, os testes de software correspondem a um grande desafio, já que os sistemas operacionais e seus softwares adicionais possuem muitas linhas de código e, em muitos casos, não são de código aberto. Para suportar esse desafio, os requisitos legais estabelecidos fornecem diretivas sólidas associadas, principalmente, a operações permitidas e perfis de acesso, segurança física, separação de software (sempre que apropriado), proteção de interfaces de comunicação, carga de software e verificação de integridade, mas não cita questões específicas como o uso de técnicas de proteção de software. Com isso, muitos fabricantes, guiados ao atendimento dos requisitos legais, não realizam a implementação de tais técnicas e deixam de incrementar os níveis de segurança de seus dispositivos.

Neste sentido, o presente trabalho apresenta uma breve discursão sobre como o uso de técnicas de proteção de software em sistemas embarcados pode auxiliar os fabricantes a melhorar a segurança e confiabilidade de seus dispositivos, incluindo aspectos relevantes para escolha da técnica apropriada e possíveis desvantagens. Para tanto, serão consideradas os principais métodos existentes na literatura, a saber, ofuscação, incorruptibilidade e marca d'água. 
O presente artigo está organizado da seguinte forma: a Seção 2 apresenta os conceitos básico de proteção de software e contextualiza a relevância de seu uso e estudo, a Seção 3 discute sobre o uso de técnicas de ofuscação em sistemas embarcados, a Seção 4 aborda as técnicas de incorruptibilidade e a Seção 5 aborda as técnicas de marca d'água. Finalmente, a Seção 6 apresenta algumas conclusões e considerações finais.

\section{Proteção de Software}

Avanços em análise de programas e tecnologias de engenharia de software têm levado ao aperfeiçoamento das ferramentas para análise e desenvolvimento seguro de software, fornecendo subsídios para que os softwares sejam mais eficientes, seguros e protegidos, o quanto possível. Estes mesmos avanços aumentam o conhecimento de adversários para a realização de engenharia reversa com o objetivo de descobrir vulnerabilidades ou realizar alterações indevidas.

As técnicas de proteção de software tem como objetivo dificultar a realização de engenharia reversa de um software, assegurar sua execução conforme esperado e rastreá-lo diante de uma possível distribuição ilegal [Collberg and Nagra 2009, Collberg and Thomborson 2002, Boccardo et al. 2010]. As técnicas de proteção de software utilizadas para tais finalidades são: ofuscação, incorruptibilidade e marca d'água. Técnicas de ofuscação de código dificultam a engenharia reversa por tornarem o código mais difícil de ser compreendido, sendo este aumento na dificuldade de compreensão caracterizado por modificações sintáticas que preservam a semântica do programa original. Técnicas de incorruptibilidade garantem que o software será executado como esperado, mesmo diante de tentativas de modificação ou monitoração por um atacante ou adversário. $\mathrm{E}$, finalmente, técnicas de marca d'água atuam como uma ferramenta de defesa contra distribuição ilegal e proteção de propriedade intelectual, por meio da embarcação de uma informação de identificação no software para torná-lo posteriormente rastreável.

Embora as técnicas de proteção de software sejam estudadas de maneira recorrente no meio acadêmico, existe pouco conhecimento entre os desenvolvedores de software sobre a importância do uso e implementação de tais técnicas. Este fato implica em um fardo ainda maior para outras medidas de contorno que visam garantir a segurança das aplicações, pois uma vez que um atacante ou adversário tenha acesso ao código do software e compreenda seu funcionamento, não há limites para o que possa fazer.

\section{Ofuscação}

Técnicas de ofuscação de código alteram a estrutura de instruções de um programa com a intenção de torná-las menos aparente, dificultando, com isso, o processo de engenharia reversa por um adversário [Collberg and Thomborson 2002, Linn and Debray 2003]. Desta forma, ofuscar um programa refere-se a produzir um novo programa semanticamente equivalente em que a extração de informações por engenharia reversa é mais difícil de ser realizada. O conceito de dificuldade está associado a quantidade de tempo, dinheiro e poder computacional adicionais gastos para efetuar a análise do programa transformado se comparado com o que seria gasto para analisar o programa original. O impacto de uma determinada transformação é mensurável de acordo com o overhead, confusão e dificuldade 
de um adversário de reverter a ofuscação [Collberg and Nagra 2009]. As transformações efetivadas por um ofuscador de código são ditadas pela quantidade de overhead que um desenvolvedor esta disposto a inserir em sua aplicação.

A ofuscação de código pode ser utilizada para prover:

- Diversidade: criação de versões distintas de um código semanticamente equivalente dificulta ataques de diferença (“diffing”) e exploração de vulnerabilidades por agentes maliciosos;

- Proteção de propriedade intelectual: o entendimento e a extração de códigos proprietários ou dados sigilosos são dificultados;

- Integridade: um código mais complicado de entender é mais complicado de ser modificado.

As técnicas de ofuscação podem dificultar as duas fases da engenharia reversa: a de disassembly ${ }^{1}$ para que esta retorne uma linguagem assembly incorreta; ou a de decompilação ${ }^{2}$ fazendo com que as ferramentas de análise estática retornem grandes aproximações, dificultando-se, assim, a recuperação da semântica do código. O processo de decompilação pode ser dificultado pela inserção de expressões booleanas complexas, ponteiros, saltos indiretos ou espúrios e aliasing $^{3}$ [Collberg and Nagra 2009].

As técnicas de ofuscação estática [Collberg et al. 2003] transformam o código do software antes de sua execução e, apesar de aumentarem o grau de dificuldade de um adversário descobrir alguma propriedade do código, as mesmas não são robustas diante de ferramentas de análise dinâmica. Já as técnicas de ofuscação dinâmica [Madou et al. 2006] transformam o código do programa em tempo de execução e, consequentemente, dificultam a tarefa de desvendar um segredo tanto por meio de análise estática quanto por análise dinâmica.

No contexto de sistemas embarcados, as técnicas de ofuscação podem ser utilizadas para dificultar que um atacante ou adversário tenha entendimento do código-fonte da aplicação e comprometa a confidencialidade do código, evitando a descoberta de possíveis vulnerabilidades, a privacidade de constantes e dados, como chaves criptográficas e dados de medição, por exemplo, e a integridade do código, uma vez que um código de difícil entendimento também torna-se difícil de ser modificado.

Dentre as técnicas de ofuscação de código disponíveis na literatura, àquelas ditas estáticas apresentam maior potencial para serem utilizadas em sistemas embarcados devido ao menor consumo de recursos computacionais se comparado com as técnicas de ofuscação dinâmica. Apesar das técnicas de ofuscação dinâmica serem mais robustas, estas apresentam uma degradação maior de desempenho devido ao fato de, geralmente, o segmento de código ser alterado, o pipeline ${ }^{4}$ de instruções percisar ser esvaziado, o conteúdo do cache de dados ter que ser escrito na memória e o cache de instruções ser invalidado.

\footnotetext{
${ }^{1} \mathrm{~A}$ fase de disassembly converte uma sequência de bits em uma linguagem assembly.

${ }^{2}$ A fase de decompilação reconstrói a estrutura semântica de um código assembly.

${ }^{3}$ Aliasing descreve uma situação em que uma posição de um dado na memória pode ser acessado através de diferentes nomes simbólicos em um programa, em que a modificação de um dado por um nome implicitamente modificaria todos os outros nomes apontados.

${ }^{4}$ Técnica de hardware que possibilita que a CPU busque uma ou mais instruções além da próxima a ser executada.
} 
Algumas técnicas de ofuscação que podem ser utilizadas incluem: transformação de layout [Collberg and Nagra 2009] que remove informações de formatação de arquivos ou substituem identificadores; tranformação de controle [Collberg et al. 2003, Majumdar et al. 2006] que distorce o fluxo de controle pela inserção de código espúrio, reordenação do fluxo de execução das instruções através de saltos condicionais/incondicionais ou eliminação das estruturas de controle (laços de repetição e instruções condicionais); e tranformação de dados [Lakhotia et al. 2005, Linn and Debray 2003] que substitue as instruções ou os dados por novas representações.

\section{Incorruptibilidade}

As técnicas de incorruptibilidade visam assegurar que um determinado programa esteja executando como esperado, mesmo em situações de modificação ou monitoração por um adversário [Collberg and Nagra 2009]. Estas técnicas além de estarem atreladas à detecção de violações de integridade, também possuem funcionalidades de resposta em situações de modificação ou monitoração.

Existe uma correlação direta entre as técnicas de incorruptibilidade e de ofuscação, pois para modificar um código é necessário que o mesmo seja entendido. Portanto, como as técnicas de ofuscação realizam transformações no código para que a engenharia reversa demande mais tempo, custo e/ou poder computacional, pode-se dizer que a aplicação de ofuscação é uma etapa que garante maior resiliência ao método de incorruptibilidade.

As técnicas de incorruptibilidade para detectar violações à integridade consistem na auto-verificação de trechos de código do programa (ou auto-verificação de hash)[Horne et al. 2002, Chang and Atallah 2002] ou na inspeção lógica do programa (através do uso de hash) [Jacob et al. 2007, Zhang and Gupta 2003]. A verificação pode ser classificada em estática, quando é feita em tempo de carregamento do programa, ou dinâmica, quando é feita durante a execução do programa. A inspeção lógica pode ser feita nos dados do programa ou no fluxo de execução. Dado que um desenvolvedor tem conhecimento a priori dos possíveis estados que seu programa pode atingir, o desenvolvedor pode inserir rotinas para verificar se uma variável ou o resultado de uma função está em conformidade com um determinado parâmetro previamente estipulado [Collberg and Nagra 2009].

Entre as estratégias comuns para a ação (resposta) de uma técnica de incorruptibilidade temos: terminar a execução do programa, restaurar o programa substituindo os trechos de código adulterados por trechos originais, retornar resultados incorretos, degradar o desempenho da aplicação, contatar o desenvolver ou até mesmo punir o sistema do adversário, por exemplo, apagando seus documentos pessoais [Gang Tan and Jakubowski 2006]. Sejam quais forem as formas de verificação e as respostas a serem tomadas, é importante que as mesmas estejam distantes em tempo (com execução não simultânea) e espaço (não próximas no código do programa) para aumentar a dificuldade de um adversário subvertê-las.

O emprego de técnicas de incorruptibilidade no projeto de sistemas embarcados pode contribuir para a realização do procedimento de verificação de integridade, por meio da verificação de trechos de código ou descarregamento do código nos casos em que exista 
diversidade de código criada por ofuscação, o que inviabilizaria o uso de resumos criptográficos pelo fato de instrumentos distintos possuirem códigos distintos. As técnicas de incorruptibilidade também podem ser usadas para verificar se o sistema embarcado apresenta o comportamento esperado, de modo que diante de um conjunto de testes funcionais estabelecidos, torna-se muito difícil para um software malicioso conseguir simular o comportamento do software original.

Os métodos de incorruptibilidade de auto-verificação de trechos de código e de inspeção da lógica de execução apresentam potencial para serem utilizados na proteção de sistemas embarcados, embora apresentem vantagens e desvantagens entre sim. Os métodos de auto-verificação de hash possuem desvantagens como a facilidade de o adversário detectar uma rotina de verificação devido a sua natureza atípica de operação, uma vez que a maioria dos programas não lêem seu próprio segmento de texto (código), além do fato destes métodos não tratarem alterações no comportamento ou dados do programa. Os métodos que inspecionam a corretude dos dados e do fluxo de controle visam contornar as deficiências dos métodos de auto-verificação de hash, mas preservam as características de acarretar uma certa degradação de desempenho e aumento de tamanho do programa devido a necessidade de cálcular o hash de uma dada região do código do programa. Entretanto, como existem diversos algoritmos para cálculo de hash que podem ser implementados de modo eficiênte (em tempo e espaço) [Aumasson 2017], ambos os métodos de incorruptibilidade podem ser adotados em sistemas embarcados.

\section{Marca d'água}

Marca d'água de software é um método de proteção normalmente utilizado contra a distribuição ilegal de software (pirataria) e/ou proteção de propriedade intelectual, no qual uma informação secreta é inserida no programa para uma posterior comprovação de sua autoria, autenticidade ou propriedade. O detentor dos direitos autorais pode estabelecer a propriedade do software extraindo a informação secreta de uma cópia - autorizada ou não - deste software.

Um esquema de marca d'água ideal teria alta taxa de dados, furtividade, resiliência e diversidade, e pode ser classificado de acordo com a técnica utilizada na extração da marca, podendo ser estático [Venkatesan et al. 2001] ou dinâmico [Collberg and Nagra 2009]. Uma marca d'água estática é inserida na área de dados (chamada de marca d'água de dados) ou no texto do código do programa (chamada de marca d'água de código). A extração de tais marcas d'água é realizada sem a necessidade de executar o programa. Já uma marca d'água dinâmica é inserida no estado de execução do programa, isto é, em um esquema de marca d'água dinâmico, a informação embarcada não corresponde a própria marca d'água, e sim a trechos de código que geram a marca d'água desejada quando o programa é executado, geralmente diante de uma entrada específica [Collberg and Nagra 2009]. Nos esquemas de marca d'água (estáticos ou dinâmicos) há usualmente quatro algoritmos:

- Codificador: converte o identificador $i d$ na marca d'água $\omega$;

- Decodificador: extrai $i d$ a partir da marca d'água $\omega$;

- Embarcador: recebe como parâmetro o programa $P$ (podendo ser o código binário ou o código-fonte em alguma linguagem de programação), a marca d'água 
$\omega$ e uma possível chave secreta $k$, e retorna como saída um programa marcado $P_{\omega}$. O embarcador preserva a semântica do programa, isto é, $P$ e $P_{\omega}$ tem o mesmo comportamento de entrada e saída;

- Extrator: retorna $\omega$ a partir de $P_{\omega}$.

Assim como para as técnicas de ofuscação e incorruptibilidade, existem na literatura diversos esquemas de marca d'água estáticos e dinâmicos, baseados em predicados opacos [Myles and Collberg 2006], alocação de registradores [Collberg and Nagra 2009], interpretação abstrata [Cousot and Cousot 2014], caminhos dinâmicos [Collberg et al. 2004] e grafos [Venkatesan et al. 2001].

As técnicas de marca d'água podem ser usadas em sistemas embarcados para verificação de integridade, por meio do reconhecimento de uma marca associada a algum comportamento de execução do dispositivo e para punir possíveis vazamentos de código por parte das instituições responsáveis pela avaliação de sistemas embarcados (acreditados pela Autoridade responsável), como o caso de alguns instrumentos de medição. Isso porque, embora a pirataria não seja uma preocupação dos fabricantes de sistemas embarcados, o roubo de propriedade intelectual é um tema crítico, já que um concorrente de posse de seu dispositivo e/ou código-fonte, pode desmonta-lo, ver como funciona, os "segredos" de projeto e, a partir dessa informação, construir um dispositivo concorrente, melhorar seu dispositivo com detalhes de projeto identificados e tomar seu mercado.

As técnicas de marcas d'água dinâmicas são mais apropriadas para o propósito de verificação de integridade, uma vez que são verificadas mediante a execuão do código. Por outro lado, as técnicas de marca d'água estáticas mostram-se mais promissoras para comprovação de autoria/propriedade, em particular marcas d'água baseadas em grafos, devido a baixa sobrecarga de tempo e espaço adicionadas ao código [Bento et al. 2017]. No que refere-se aos cenários de ataque, as marcas d'água dinâmicas são mais resistentes diante de ataques de falsificação, remoção ou adição, já que estão associadas a um estado dinâmico do programa que, consequentemente, não são estaticamente visíveis para um adversário.

\section{Conclusão}

O presente trabalho apresentou considerações sobre técnicas de ofuscação, incorruptibilidade e marca d'água, e vantagens de adotá-las em projetos de sistemas embarcados. É importante observar que mesmo com os recursos computacionais reduzidos de tais dispositivos ainda existem técnicas de cada tipo apresentado que são facilmente aplicáveis com degradação de desempenho e/ou aumento no tamanho do código final não proibitivos.

Embora as técnicas de proteção de software sejam abordadas em muitos trabalhos de maneira isolada, a utilização em conjunto destas técnicas corresponde a uma boa prática para aumentar a confiança de que o propósito buscado com o uso de cada uma destas está sendo alcançado corretamente. Note que o uso de técnicas de marca d'água para verificação de integridade e/ou proteção de propriedade intelectual é complementada pelo uso de técnicas de incorruptibilidade, que podem visar garantir, além dos aspectos apresentados ao longo do texto, a não adulteração da marca d'água, e a ofuscação, por sua vez, auxilia na ocultação do uso das técnicas de marca d'água e incorruptibilidade. 
O estudo detalhado de quais técnicas de proteção de software são adequadas aos dispositivos de software embarcado, o impacto na adotação de tais técnicas no projeto destes dispositivos, e como e quais requisitos legais podem ser atendidos com o uso de tais técnicas serão investigados em trabalhos futuros.

\section{Referências}

Aumasson, J.-P. (2017). Serious Cryptography: A Practical Introduction to Modern Encryption. No Starch Press, San Francisco, CA, USA.

Bento, L. M. S., Boccardo, D. R., Machado, R. C. S., Pereira de Sá, V. G., and Szwarcfiter, J. L. (2017). Marca d'água estruturada. In XVII Simpósio Brasileiro de Segurança da Informação e de Sistemas Computacionais - SBSeg 2017, pages 388-399, Brasília-DF.

Boccardo, D. R., Machado, R. C. S., and Carmo, L. F. R. C. (2010). Tranformações de código para proteção de software. In Minicurso SBSEG 2010, Fortaleza-CE: Sociedade Brasileira de Computação.

Chang, H. and Atallah, M. J. (2002). Protecting software code by guards. In Sander, T., editor, Security and Privacy in Digital Rights Management, pages 160-175, Berlin, Heidelberg. Springer Berlin Heidelberg.

Chelf, B. (2011). Measuring software quality — a study of open source software. In Chief Technology Officer. Coverity.

Collberg, C., Carter, E., Debray, S., Huntwork, A., Kececioglu, J., Linn, C., and Stepp, M. (2004). Dynamic path-based software watermarking. In Proceedings of the ACM SIGPLAN 2004 Conference on Programming Language Design and Implementation, PLDI '04, pages 107-118, New York, NY, USA. ACM.

Collberg, C. and Nagra, J. (2009). Surreptitious Software: Obfuscation, Watermarking, and Tamperproofing for Software Protection. Addison-Wesley Professional, 1st edition.

Collberg, C. S. and Thomborson, C. D. (2002). Watermarking, tamper-proofing, and obfuscation - tools for software protection. IEEE Trans. Software Eng., 28(8):735746.

Collberg, C. S., Thomborson, C. D., and Low, D. W. K. (2003). Obfuscation techniques for enhancing software security. US6668325B1.

Cousot, P. and Cousot, R. (2014). Abstract interpretation: Past, present and future. In Proceedings of the Joint Meeting of the Twenty-Third EACSL Annual Conference on Computer Science Logic (CSL) and the Twenty-Ninth Annual ACM/IEEE Symposium on Logic in Computer Science (LICS), CSL-LICS '14, pages 2:1-2:10, New York, NY, USA. ACM.

Gang Tan, Y. C. and Jakubowski, M. H. (2006). Delayed and controlled failures in tamperresistant systems. In The 8th Information Hiding Conference, Alexandria, VA.

Horne, B., Matheson, L., Sheehan, C., and Tarjan, R. E. (2002). Dynamic self-checking techniques for improved tamper resistance. In Sander, T., editor, Security and Privacy in Digital Rights Management, pages 141-159, Berlin, Heidelberg. Springer Berlin Heidelberg. 
IDC, I. D. C. (2014). Final study report: Design of future embedded systems (smart 2009/0063). Technical report, Information Society and Media of the European Commission.

Insider, B. (2018). Former cisco ceo john chambers predicts 500 billion connected devices by 2025 . urlhttps://www.businessinsider.com/former-cisco-ceo-500-billionconnected-devices-by-2025-2015-11?utm_source=feedly\&utm_medium=referral.

Jacob, M., Jakubowski, M. H., and Venkatesan, R. (2007). Towards integral binary execution: Implementing oblivious hashing using overlapped instruction encodings. In Proceedings of the 9th Workshop on Multimedia \& Security, MM\&\#38;Sec '07, pages 129-140, New York, NY, USA. ACM.

Lakhotia, A., Kumar, E. U., and Venable, M. (2005). A method for detecting obfuscated calls in malicious binaries. IEEE Transactions on Software Engineering, 31(11):955968.

Linn, C. and Debray, S. (2003). Obfuscation of executable code to improve resistance to static disassembly. In Proceedings of the 10th ACM Conference on Computer and Communications Security, CCS'03, pages 290-299, New York, NY.

Madou, M., Anckaert, B., Moseley, P., Debray, S., De Sutter, B., and De Bosschere, K. (2006). Software protection through dynamic code mutation. In Song, J.-S., Kwon, T., and Yung, M., editors, Information Security Applications, pages 194-206, Berlin, Heidelberg. Springer Berlin Heidelberg.

Majumdar, A., Thomborson, C., and Drape, S. (2006). A survey of control-flow obfuscations. In Bagchi, A. and Atluri, V., editors, Information Systems Security, pages 353-356, Berlin, Heidelberg. Springer Berlin Heidelberg.

Myles, G. and Collberg, C. (2006). Software watermarking via opaque predicates: Implementation, analysis, and attacks. Electronic Commerce Research, 6(2):155-171.

Venkatesan, R., Vazirani, V., and Sinha, S. (2001). A graph theoretic approach to software watermarking. In Moskowitz, I. S., editor, Information Hiding, pages 157-168, Berlin, Heidelberg. Springer Berlin Heidelberg.

Zhang, X. and Gupta, R. (2003). Hiding program slices for software security. In Proceedings of the International Symposium on Code Generation and Optimization: Feedback-directed and Runtime Optimization, CGO '03, pages 325-336, Washington, DC, USA. IEEE Computer Society. 